\title{
The Newcastle NHS Chronic Fatigue Syndrome Service: not all fatigue is the same
}

\author{
${ }^{1} \mathrm{JLN}$ Newton, ${ }^{2} \mathrm{H}$ Mabillard, ${ }^{3} \mathrm{~A}$ Scott, ${ }^{4} \mathrm{~A}$ Hoad, ${ }^{5} \mathrm{G}$ Spickett \\ ${ }^{1}$ Professor of Ageing and Medicine, NIHR Biomedical Research Centre in Ageing and Institute for Ageing \& Health, Newcastle University, and \\ Consultant Physician, Northern Regional CFS/ME Clinical Service, Newcastle upon Tyne Hospitals NHS Trust; ${ }^{2,3}$ Medical students, NIHR \\ Biomedical Research Centre in Ageing and Institute for Ageing \& Health, Newcastle University; ${ }^{4}$ Foundation doctor; ${ }^{5}$ Consultant Immunologist and \\ Northern Regional CFS Clinical Service Champion, Northern Regional CFS/ME Clinical Service, Newcastle upon Tyne Hospitals NHS Trust, UK
}

\begin{abstract}
In England the Department of Health has funded specialist clinical services aimed at diagnosing and managing the symptoms of chronic fatigue syndrome (CFS). These services are not available to those who do not fulfil the diagnostic criteria for CFS. This service evaluation examined the proportion of those referred to a specialist CFS service fulfilling the Fukuda diagnostic criteria for CFS and the alternative fatigue-associated diagnoses. The CFS database was interrogated to include every patient referred to the Newcastle service from November 2008 to December 2009. All medical notes were reviewed and the diagnosis, sex and age recorded. Data were compared to a previous service evaluation (2005-07). In 2008-09, 260 subjects were referred: 19 referrals per month (260/I4), compared with 17 referrals per month in 2005-07 (375/24). The proportion of patients diagnosed with CFS increased significantly compared with 2007 (36\% [20/56] vs 60\% [157/260]; $p<0.000$ I). Of the $40 \%$ of patients subsequently found not to have CFS the most common diagnosis was fatigue associated with a chronic disease (47\% of all alternative diagnoses); $20 \%$ had primary sleep disorders, 15\% psychological/psychiatric illnesses and $4 \%$ a cardiovascular disorder. Thirteen per cent remained unexplained $(5.2 \%$ of the total referrals). This study found a significant increase in the proportion of patients referred to National Health Service (NHS) CFS services diagnosed with CFS. A large proportion of patients presenting with fatigue are not eligible for referral to the Department of Health specialist fatigue services, which represents an unmet need in terms of symptom management in current NHS services.
\end{abstract}

KEYWORDS Chronic disease, chronic fatigue syndrome, fatigue

DECLARATION OF INTERESTS The cohort study was funded by the United Kingdom NIHR Biomedical Research Centre in Ageing - Cardiovascular Theme, ME Research UK, John Richardson Research Group and the Irish ME Trust.
Correspondence to JL Newton, NIHR Biomedical Research Centre in Ageing and Institute for Ageing \& Health, Newcastle University, Newcastle upon Tyne NEI 4LP, UK

tel. $+44(0) 1912824128$

e-mail julia.newton@nuth.nhs.uk

\section{INTRODUCTION}

Fatigue is a debilitating symptom that affects all age groups and can impact on patients' quality of life. It is now recognised that the symptom of fatigue can occur in association with a wide range of chronic diseases, can be unexplained (chronic fatigue or idiopathic fatigue) or can occur in conjunction with a constellation of symptoms that form the chronic fatigue syndrome (CFS). A recent study found that $25 \%$ of all general practitioner (GP) consultations were characterised by the symptom of fatigue; in $6.5 \%$ of consultations fatigue was the main reason for GP attendance.'

In 2006, the UK's National Institute for Health and Clinical Excellence (NICE) produced guidelines recommending that patients who fulfil the diagnostic criteria for CFS be referred to a specialist CFS clinical service. ${ }^{2}$ The Department of Health (DOH) consequently funded clinical services aimed at diagnosing and managing the symptoms of CFS. However, these services are not available to those who do not fulfil the diagnostic criteria for CFS.

Chronic fatigue syndrome is a clinical condition characterised by disabling fatigue in conjunction with a constellation of symptoms, including musculoskeletal pain, poor memory and concentration and sleep disturbance. $^{3-5}$ It affects all age groups and is associated with significant functional impairment. ${ }^{6}$ Currently, there is no diagnostic test for CFS, making it frequently a diagnosis of exclusion, which is dependent on ensuring fatigued patients do not have another fatigue-associated diagnosis. Although the NICE guidelines prompt the exclusion of a number of such conditions, as medical knowledge and recognition of fatigue in association with chronic diseases improves there is the concern that without increased education, referrers to CFS services will not recognise the 'newer' fatigue-associated diseases and that the proportion of those referred to the DOHfunded CFS services who do not fulfil the diagnostic criteria for CFS will increase. 


\section{METHODOLOGY}

All referrals made to the Newcastle CFS Clinical Service are entered into a database. The CFS database was interrogated to include every patient referred to the service from November 2008 to December 2009. All patients who did not attend their appointments (resulting in no diagnosis being made by our service) were excluded. After these exclusions, 260 patients were seen by the service during the 14-month period. All medical notes for each patient were reviewed and the diagnosis, sex and age recorded. Results were compared with a previous small service audit performed in 2007. In this previous, limited audit, all referrals made to the service between January 2005 and December 2007 were reviewed. A random sample of 56 patients' notes was reviewed from the 375 patients seen during that period.

The UK national minimum dataset uses the Fukuda diagnostic criteria for CFS. ${ }^{7}$ Those whose symptoms matched the criteria were considered to have CFS. Within the CFS group we considered three particular clinical phenotypes:

- $\quad$ Positional orthostatic tachycardia syndrome (POTS). This is a recently recognised association with CFS. 8.9 Its diagnosis is made when patients experience symptoms on standing in association with a rise in heart rate to above 120 beats per minute (bpm) or by 30 bpm;

- CFS/fibromyalgia overlap;

- Those who described their symptoms as being precipitated by a viral or bacterial infection.

Where subjects did not fulfil the Fukuda criteria for CFS, their diagnoses were classified into:

- Fatigue associated with chronic disease;

- Primary sleep disorder;

- Cardiovascular disorder;

- Psychological/psychiatric illness;

- Idiopathic fatigue; and

- Other.

The Newcastle CFS Service has close links with the local sleep service and cardiovascular testing. Referrals to these services are arranged as appropriate according to patients' presenting symptoms.

\section{Analysis}

All data were normally distributed. Comparisons were therefore made between the proportions in each group using Fisher's exact test $(F)$ and between continuous variables using the Student's unpaired t-test $(T)$. Results were considered to be significant at the $p=0.05$ level.

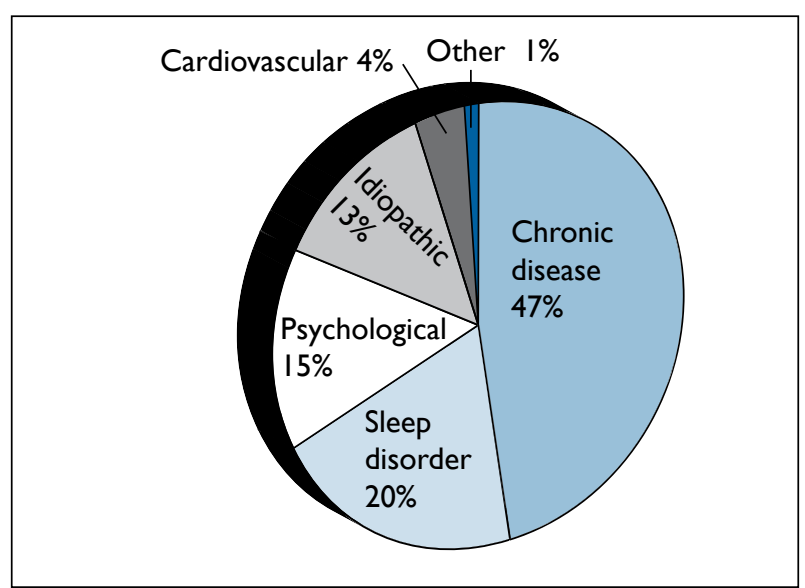

FIGURE I The diagnostic categories of patients who did not meet the criteria for CFS and were found to have an alternative diagnosis by our service in 2008-9.

\section{Approvals}

Review by the local research and development department considered that this was a service evaluation. Data storage was approved by the Caldicott guardian and the data protection officer.

\section{RESULTS}

In the 2008-09 period 260 patients were referred to our clinical service, which equated to approximately 19 referrals per month (260/14); this compared with 17 referrals per month in 2005-07 (375/24). The proportion of patients diagnosed with CFS by the Newcastle service has increased significantly compared with the results of our previous audit in $2007(36 \%$ [20/56] vs 60\% [I57/260]; F $p<0.000 I)$.

In the 2008-09 cohort, there were no significant age differences between those found to have CFS and those found to have other fatigue-associated diseases (nonCFS 41 years \pm 13 vs CFS 41 years $\pm 14 ; T p=1.0$ ) and the proportion of males was significantly lower in the group ultimately diagnosed with CFS (non-CFS 34/103 [33\%] vs 33/I57 [21\%]; F $p=0.05)$.

In the CFS group, four patients were found to have a CFS/POTS phenotype (3\%), 3I had CFS precipitated by an infective episode (20\%) and four had a CFS/ fibromyalgia phenotype (3\%).

Forty per cent of patients seen by our service were subsequently found not to have CFS. Their relative proportions and specific diagnostic groups are shown in Figure I and Table I. The most common diagnosis in these patients was fatigue associated with a chronic disease, $47 \%$ of all alternative diagnoses. This was followed by primary sleep disorders (20\%), which included eight patients with obstructive sleep apnoea (OSA), 12 with another primary sleep disorder, one patient with restless leg syndrome (RLS) and one patient 
TABLE I Other fatigue-associated diseases found in patients attending the Newcastle CFS Service

\begin{tabular}{|c|c|}
\hline Disease & Number \\
\hline Metabolic syndrome & 8 \\
\hline Neurological disorder & 13 \\
\hline Connective tissue disorder/autoimmune disease & 9 \\
\hline Medications & 1 \\
\hline Pain & 3 \\
\hline Fibromyalgia & 5 \\
\hline Coeliac disease & 2 \\
\hline Overtraining syndrome & 1 \\
\hline Cancer & 1 \\
\hline Others: & \\
\hline Low body mass index & I \\
\hline Haemochromatosis & I \\
\hline Microprolactinoma & I \\
\hline Lyme disease & I \\
\hline Immunocompromised & I \\
\hline
\end{tabular}

with both RLS and OSA. Fifteen per cent of all alternative diagnoses were psychological/psychiatric illnesses, and $4 \%$ were cardiovascular disorders. In 13\% of these patients the cause for fatigue remained unexplained ( $5.2 \%$ of total referrals).

The conditions most commonly seen in the group that had a psychological illness contributing to their fatigue were depression, anxiety and post-traumatic stress disorder. The cardiovascular diagnoses were vasovagal syncope in patients with fatigue symptoms who also had a history of episodes of loss of consciousness. The diagnosis was made after a reproduction of symptoms in head-up tilt testing.

\section{DISCUSSION}

This study has shown a significant increase over the past four years in the proportion of patients referred by GPs to an NHS CFS service who were subsequently diagnosed with CFS. This may be due to an improved recognition of CFS by GPs or hospital doctors due to better training or increased awareness of the guidelines. Alternatively it may be related to an increased awareness of fatigue-associated chronic diseases, which could lead to increased recognition and diagnosis by GPs and hospital doctors and referrals to specialty-specific clinics rather than a CFS clinic.

It is a matter for debate whether this pathway of care for the management of fatigue in those who have a chronic disease is appropriate. The focus of management in the majority of chronic disease clinics is often on monitoring the progression of the underlying disease. However, studies exploring the aetiology of fatigue in a number of chronic diseases have shown that the fatigue experienced by patients is unrelated to the underlying disease severity, ${ }^{10-13}$ with the implication that improving chronic disease management may not lead to improvements in the symptoms experienced by patients.

It is becoming evident that fatigue is a generic symptom that affects large numbers of patients and that perceived fatigue is comparable across different diseases; management strategies that work for fatigue in one disease may therefore also be appropriate in others. ${ }^{14}$ However, in the NHS in England and Wales only patients with fatigue who fulfil the Fukuda criteria for CFS can currently access specialist fatigue management services.

Our work suggests that large numbers of patients with fatigue are unable to access these specialist services and we believe that the current NHS has nothing to offer in the way of evidence-based management for this patient group. We would suggest that, like pain (where pain clinics have developed over the past decade), fatigue is a generic symptom, the management of which is not always appropriately met by the disease-specific chronic disease clinics typical of current NHS practice.

In Europe, the model of care is centred on the symptom of fatigue rather than the underlying disease process, with established fatigue management clinics effectively managing this symptom. As in Europe, we believe that fatigue is an important symptom, leading to functional impairment and significantly affecting patients' quality of life, and that developing an appropriate evidence base to optimise its management is vital if improvements in chronic disease management are to be realised.

Our patients with alternative diagnoses to CFS showed some interesting trends. The largest proportion had fatigue related to chronic diseases, most commonly connective tissue diseases, diabetes mellitus, nonalcoholic fatty liver disease and chronic lung diseases. This finding emphasises how important it is to be aware that these diseases are increasingly being recognised as associated with fatigue and that considering this in a holistic assessment of patients will optimise outcomes.

Moreover, one fifth of our patients with alternative fatigue-associated diseases were diagnosed with sleep disorders - an important, potentially treatable diagnostic group. Fatigue services therefore need to ensure effective links with sleep clinics.

In conclusion, we suggest that referral networks are established for fatigue evaluation and management, specifically for departments that commonly manage chronic diseases. 


\section{REFERENCES}

I Cullen W, Kearney Y, Bury G. Prevalence of fatigue in general practice. Ir J Med Sci 2002; I7I:I0-2. doi:I0.1007/BF03 |6893 I

2 National Institute for Health and Clinical Excellence. Chronic fatigue syndrome/myalgic encephalomyelitis (or encephalopathy). London: NICE; 2007. Available from: http://www.nice.org.uk/ nicemedia/pdf/CG53QuickRefGuide.pdf

3 Fernández AA, Martín AP, Martínez MI et al. Chronic fatigue syndrome: aetiology, diagnosis and treatment. BMC Psychiatry 2009 9: SI-II. doi:I0.I I86/I47I-244X-9-SI-SI

4 CFS/ME Working Group. A report of the CFS/ME Working Group. London: Department of Health; 2002. Available from: http://www dh.gov.uk/en/Publicationsandstatistics/Publications/Publications PolicyAndGuidance/DH_4064840

5 Prins JB, van der Meer JWM, Bleijenberg G. Chronic fatigue syndrome. Lancet 2006; 367:346-55. doi:I0.10I6/SOI40-6736(06)68073-2

6 Costigan A, Elliott C, McDonald C et al. Orthostatic symptoms predict functional capacity in chronic fatigue syndrome: implications for management. QJM 2010; 103:589-95.

7 Fukuda K, Straus SE, Hickie I et al. The International Chronic Fatigue Syndrome Study Group. The chronic fatigue syndrome: a comprehensive approach to its definition and study. Ann Intern Med 1994; 121:953-9.
8 syndrome is an under-recognised condition in chronic fatigue syndrome. QJM 2008; 101:96I-5.

9 Ewan V, Norton M, Newton JL. Symptom improvement in postural orthostatic tachycardia syndrome with the sinus node blocker Ivabradine. Europace 2007; 9:1202. doi: 10.1093/europace/eum235

10 Newton JL, Bhala N, Burt J et al. Characterisation of the associations and impact of symptoms in primary biliary cirrhosis using a disease specific quality of life measure. J Hepatol 2006; 44: 776-83. doi:I0.1016/j.jhep.2005.12.012

I I Legge $\mathrm{H}$, Norton M, Newton JL. Fatigue is significant in vasovagal syncope and its associated autonomic symptoms. Europace 2008; 10:1095-101. doi:10.1093/europace/eun 164

12 Newton JL, Jones DE, Henderson E et al. Fatigue in non-alcoholic fatty liver disease (NAFLD) is significant and associates with inactivity and excessive daytime sleepiness but not with liver disease severity or insulin resistance. Gut 2008; 57:807-I3.

I3 Newton JL, Brown A, Jones DE et al. Fatigue in early renal disease. Br J Renal Med 2009; 14:10-4.

14 Jones DE, Gray JC, Newton JL. Perceived fatigue is comparable between different disease groups. QJM 2009; 102:617-24.

\section{Exclusive offer for RCPE Collegiate Members $\mathbf{5 0 \%}$ discount on Fellowship subscriptions}

We are pleased to offer a significant discount in annual subscription rates for eligible Collegiate Members who are Consultants and wish to progress to Fellowship. ${ }^{*}$ Collegiate Members of four years' standing who are successful in their nomination for Fellowship will be able to obtain a $50 \%$ discount on their first year's Fellowship subscription and a $25 \%$ reduction on their second year's subscription, offering savings of up to $£ 300$.

Please access details of our reduced subscription rates, including concessionary elements for Fellows working less than halftime or on maternity leave, at: www.rcpe.ac.uk/join/ fellowshipoffer.php

\section{Fellowship confers a range of additional} benefits and opportunities:

- International peer and public recognition through the use of the 'FRCP Edin' postnominals.

- Professional support for revalidation/ recertification.

- The opportunity to participate in projects and working groups to determine the future direction of education and clinical medicine.

- The opportunity to help maintain national and international clinical standards by acting as an MRCP(UK) examiner.

- The opportunity to inform College responses to external policy consultations in your specialty.

- The opportunity to participate in the governance of the College through election to committees and to Council.

*Eligible candidates should normally have held a substantive Consultant post or equivalent for at least 11 months. If you hold such a post and are interested in being considered for Fellowship, the principal method is nomination by an existing Fellow. Please e-mail Avril Harries at a.harries@rcpe.ac.uk for a list of Fellows in your area. Alternatively, you may wish to discuss nomination with one of our Regional Advisers or consider self-nomination. 